\title{
Satellite Communications for Emergency
}

\author{
Enrico Del Re \\ Department of Electronics and Telecommunications \\ University of Florence and CNIT \\ Florence, Italy
}

\begin{abstract}
This paper aims at defining the role of a satellite component in an integrated communications, localization and monitoring system, which can be used in all emergency management phases. Particular attention will be given to the integration of the satellite and terrestrial components and of the different functionalities.
\end{abstract}

Keywords: Integrated satellite-terrestrial system, Communications, Localization, Monitoring, Emergency Management, Cognitive Networks.

\section{INTRODUCTION}

The role of a satellite system is fundamental in emergency scenarios, where in many cases it represents the unique immediate available resource. Nowadays the satellite system can play not only the traditional role of communications as it can add two additional essential functionalities of navigation/positioning and monitoring/sensing.

The huge attention paid by international entities, governments, scientific community and operators devoted to security and safety topics emphasizes the requirements of having complete functionalities, high response speed and adaptation capabilities to differentiated contexts. Efficient and complete emergency systems can be provided by the synergistic use of communication, positioning and global monitoring services: this trend is confirmed by the initiatives of security and safety agencies, research institutions, standardization organizations and by the European Commission guidelines [1-7]. This paper proposes an integrated communication, localization and monitoring system, which can be used in all phases of the emergency management. Particular attention will be given to the integration among networks and functionalities, generally conceived as stand-alone and not necessarily oriented to multi-disciplinary applications. Specific topics that will be addressed are:

- the methods of collection, analysis and integration of pre- and post-event GMES data, the data fusion techniques among the information coming from earth; - observation systems and the information collected by sensors deployed on site;

- the context evaluation through cognitive approaches in order to identify the best transmission strategies, taking into account the scarcity of resources in an emergency context;

- advanced localization techniques which are based on the use of Global Navigation Satellite Systems (GNSS) assisted by available positioning information, which can be derived from both the communication and monitoring networks.

In this framework, increased performance can be achieved through the adoption of an adaptive cooperative approach based on communication among users, communication network, emergency control and coordinator center and HAPs or mini-satellites. The emergency systems here considered are characterized by meshed heterogeneous architectures based on both satellite and terrestrial segments: in particular, the use of survived networks, which are involved in the disaster but still partially operating and networks deployed after the critical event in the intervention area is assumed. Alerting and control signaling are defined according to opportunistic, cognitive and cooperative paradigms. Satellite and terrestrial components will be defined, including a distributed swarm of mini-satellites, and the definition of terrestrial infrastructure and communication protocols which are required for the transmission of the localization and monitoring information, collected with frequency and reliability needed for an emergency scenario in the different management phases. The integration of sensors and terminals (even wearable) with other emergency networks represents another important issue: these devices can transmit biometric information, data, images also in data fusion procedures on the involved area and through cooperative interactions among the devices themselves. In emergency conditions, especially during the intervention and mitigation phases, the availability of NAV/COM/GMES devices lead to remarkable gains in terms of speed of response, completeness and effectiveness of intervention. These benefits can be completely exploited using reconfigurable and interoperable systems because of the different networks in the considered scenario. The final objective is to describe feasible integration of:

- high reconfigurable communication technologies;

- $\quad$ assisted satellite-terrestrial localization technologies;

- information management technologies, adopted on information coming from GMES data fusion operations.

Nowadays no existing systems integrate these three functionalities.

\section{SYSTEM ARCHITECTURE}

The whole management cycle of an emergency 
situation has to be taken into account, aiming at defining a set of technologies that could be included in a service platform in order to allow a timely, complete and more decisive response, to improve the organization for analog events and develop a faster and more operative alert and response capabilities. The management of emergency situation is composed of four different phases, shown in Figure 1:

- Preparedness: preparation in terms of planning, training of people, updating of solutions on the basis of experience and information learned during the different phases of past emergencies;

- Response: both as alert to catastrophic event and as activation of control center for appropriate intervention on the territory, and as the First Responder intervention to minimize the effects;

- Recovery: as completion of rescue actions and reestablishment of normal conditions;

- Mitigation: the mitigation of consequence through evaluation of measures to be implemented to prevent other disasters and to secure the disaster area.

The synergistic use of communication, positioning and global monitoring services, provided by means of meshed heterogeneous architectures based on satellite and terrestrial segments, resorting to the most recent cognitive, cooperative and context- and

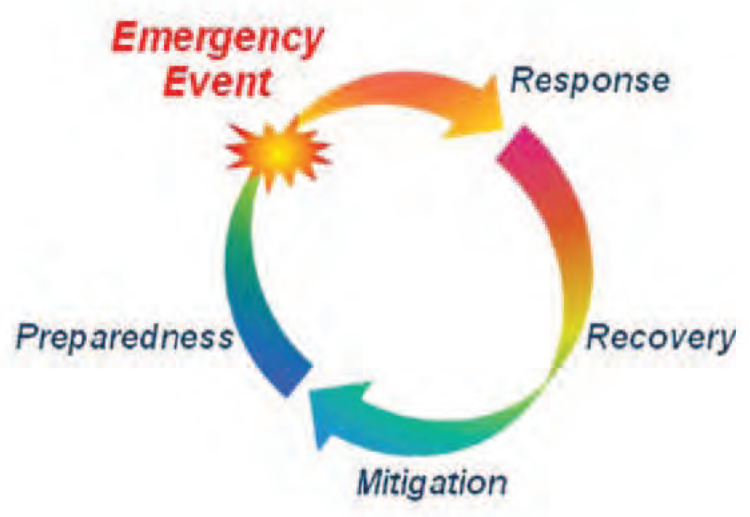

Figure 1 - Emergency management phases

location-aware technologies, with deterministic and opportunistic radio access, can afford remarkable gains in terms of effectiveness, speed of response and performance in all phases of emergency situation management. This paper proposes a future integrated system integrating the three functionalities of communication, navigation/positioning and monitoring/sensing, identifying the possible improvements and the adaptation to different emergency contexts that characterize this services platform, including interoperability and scalability. To this end it is necessary to consider different and heterogeneous contributions coming from the NAV, COM and GMES domains, to define their synergistic integration and cooperative use, and foresee the reconfiguration capability of devices and infrastructure.
The proposed architecture (Figure 2) is intrinsically heterogeneous and hybrid, able to keep the interaction between partially or totally survived networks and network deployed on the emergency scenario to provide for coverage holes: in this architecture the satellite infrastructure plays a leading role for its independence from the catastrophic event as well as for its ability of collecting information created by sensors deployed on the territory. In the framework of communication architecture also solutions based on mini-satellites networks are of interest. The use of satellite and terrestrial segments can provide GMES system functionality: this system covers the issues of monitoring, security and safety information, through the collection of information via satellite and in-situ and the harmonization capability of data; the benefits coming from the introduction of mini-satellites solution (considered as in-situ data acquisition center) can provide additional improvements. The considered architecture makes possible an assisted localization, through the integration of GPS information, available for outdoor scenarios and the information received from other in-situ terminals.

As far as the communication subsystem is concerned, assuming that pre-existing (to disaster) communication networks are totally or partially unavailable, the overall system architecture provides a cognitive approach that, due to spectrum sensing techniques, highlights spectral "holes" and uses them in opportunistic ways to activate emergency links. In particular it is able to provide techniques that use survived and emergency network resources in cognitive and cooperative modes to facilitate the alarm signaling diffusion and to allow an optimal management of rescue phases. Communications allow the diffusion over the IAN (Incident Area Network) of both geographic images and information of different nature. The system also includes sensors, terminals and interfaces useful for emergencies: these devices aim at the acquisition and integration of biometric and geographic data acquired in different places on the territory and should be highly reconfigurable due to the high number of systems that could have survived to the catastrophic event.

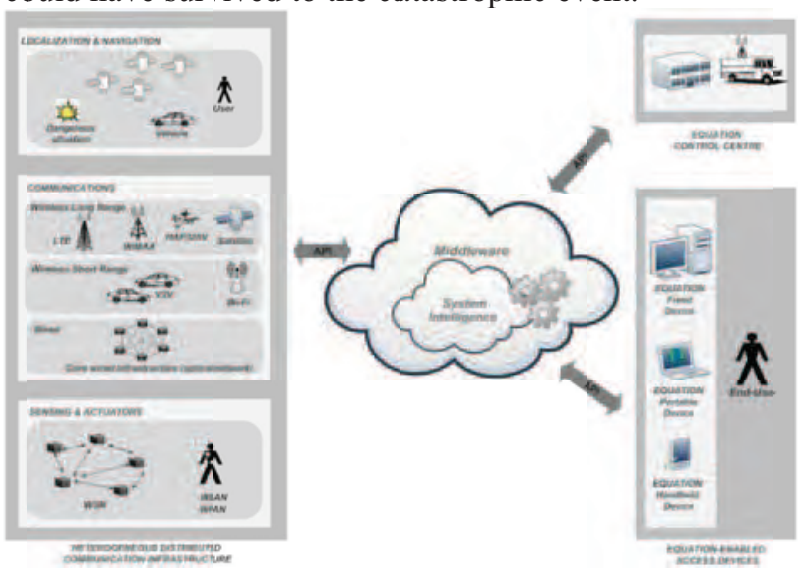

Figure 2-A possible system architecture 
They should include advanced localization functionality able to manage adaptive use of resources and transport on multiple channels.

The integration of the three components and of the novel technologies previously described determines benefits in every phases of the emergency management:

- Response: communication, cooperation and cognitive techniques in hybrid systems, data acquisition on field and transmission to control center, alarm signaling via stand-alone and survived networks;

- Recovery: opportunistic communications and localizations techniques, sending images and information on field, use of mini-satellites (swarm), definition of architecture more oriented to resource responsiveness and optimization;

- Mitigation: on line data fusion techniques via "object" sensors that can allow to reduce damages after alarm and first response phases;

- Preparedness: training first responder, definition of features that allow services platform to adapt to different scenarios, utilization of GMES together with information acquired during the emergency.

\section{ADVANCES BEYOND STATE-OF-THE-ART}

Presently no emergency system integrates the three features of communication, localization and monitoring. In international and national contexts there are systems that offer one or two of these solutions. The European recommendation published by ETSI, in the EMTEL program, has recognized the importance of "Location Services" to provide real time information about people and vehicles position to a control center [1]. This document is one of the pillar at the basis of many research and development enterprises to achieve networking and communication integrated system: among the others, ESS (Emergency Support System) [2], SAFER (Services and Applications for Emergency Response) [3], WISECOM (Wireless Infrastructure over Satellite for Emergency Communications) [4], CHORIST (Integrating Communications for Enhanced Environmental Risk Management and Citizens Safety) [5]. In national scenarios, the SALICE project (Satellite-Assisted Localization and Communication for Emergency services) [6-7] attained important results for the management of emergency events, improvement of response team capability, and networking and communication integration in a single infrastructure via SDR technology.

An updated state of the art of satellite communication and navigation systems can be found in [8].

Compared to the current state of art, this paper proposes an architectural and technological enhancement through integration of GMES information in emergency NAV/COM system, integration of nano-satellites in hybrid terrestrial/satellite network infrastructure, the localization in absence of a sufficient number of LOS satellites, the cooperation between sensors and communication network, the adoption of cognitive approach in emergency systems, the reconfigurable BTS implementation. Briefly, it proposes a NAV/COM/GMES integrated system able to provide the necessary information in emergency scenarios in a complete, reliable and flexible mode. This system is able to provide the three features required in future call of Theme 9 (Space) of FP7 (SPA.2009.1.1.03: Integration of SatCom /SatNav with GMES for prevention and management of emergencies).

\section{A. Integration of GMES into NAV/COM emergency systems.}

Decision-making ability in critical emergency situations is strengthened by the availability of information on the effects on the territory following a catastrophic event. Among the observation and monitoring systems, we underline the exploitation of Earth Observation data and techniques generically collected under the GMES (Global Monitoring for Environment and Security) [910]. GMES is an initiative of the UE aiming to provide an integrated set of updated information about the environment at different spatial and temporal scales exploiting earth observation systems as well as other methods to collect environmental data. A global view of GMES comprises remote earth observation systems based on satellites, aerial or maritime platforms, in-situ sensors, data processing centers and techniques integrating collected data and ground information systems, maps, local data. The resulting information must be then transferred in aggregated and meaningful form to the final users, i.e. the command center and the operating teams for their specific needs. Early response to disaster events can be eased by satellite remote sensing, as a useful instrument to help the decision chain by the civil protection authorities [11-12]: therefore it is clear the importance of developing the GMES component in the future emergency system and the central role devoted to it under the 7th Framework Programme of the European Commission.

\section{B. Integration of nano-satellites in the hybrid terrestrial satellite network infrastructure.}

The integration and distribution of data from in-situ sensors and images obtained from satellite has remarkable possibility of improvement: this aspect, very important for emergency management in order to save lives and preserve the territory, does not meet the needs of speed and accuracy. For example it is important to understand if a fire is heading towards towns, and thus understand to which give priority in the management of available resource to face the emergency situation. Current systems are inadequate to follow the evolution of a phenomenon that evolves rapidly both in terms of spatial resolution $(4-5 \mathrm{~km}$ at Italian latitudes) and revisit times (6-8 hours). Using a constellation of "several" small (and low cost) satellites could provide a better trade-off for spatial resolution/revisit time. Moreover, the use of constellations of very small and low cost satellites is 
emerging also in the telecommunications field: they could be used to "virtually" replace a big satellite or as communication relays for big satellites [13-14]. Minisatellites with mass lower than $100 \mathrm{~kg}$ (including also nano-satellites with weight lower than $50 \mathrm{~kg}$ and picosatellites with mass lower than $10 \mathrm{~kg}$ ) allow to have development and launch costs of some orders of magnitude lower than those relative to medium/big satellites (with mass higher than $500 \mathrm{~kg}$ ). The disadvantages derived from use of mini satellites are represented by reduced available resources on board of satellite both at the payload level (NAV/COM/GMES services capability) and at the platform level (attitude control, orbital control and generated power).

\section{Localization in emergency scenarios.}

The use of GNSS-based positioning in an emergency scenario may yield several advantages. GPS equipped terminals are widely used and even their modernization for effective management of rescue operations has been studied [15-16]. The topic of hybrid localization (considering in particular the synergy between satellite navigation and communications) is not new in the scientific literature. However, the state of development of the research in this field has not yet brought the techniques to such a consolidated state that allows an effective use; several aspects have still to be addressed and refined. In the particular scenario considered we may take advantage from a cooperative approach to the localization, by means of interaction among the different user terminals, between the user terminal and the communication network, between the terminal and a control center for the management of the operations. Recently in the scientific literature a more complex approach is under investigation, taking advantage not only of a single assistance station, but exploiting also the partial positioning information distributed among the users that are within a certain area. This kind of architecture is referred as cooperative positioning or peer-to-peer (P2P) positioning. The paradigm of P2P localization relies on the possibility to exploit the existence of direct communication links among nodes of a network, generally equipped with GNSS receivers, to transmit collaboration data thus enabling determination of nodes location anytime and anywhere. It is well known that GNSS systems provide highly reliable positioning when at least four satellites are visible at the node receiver antenna. This is generally guaranteed in open sky outdoor environments. On the contrary, in scenarios where the line-of-sight (LOS) between satellites and receiver's antenna is obstructed, GNSS-based localization heavily degrades or completely fails. Under these circumstances other localization techniques based on the concept of collaboration have to be considered. For example in [18] the collaboration concept is widely addressed for a vehicular environment, also resorting to the integration of different technologies (GPS and INS). It is worth noting that literature on P2P is, however, still limited.
D. Cooperation and opportunistic techniques in emergency networks.

Cooperation is a novel communication paradigm in wireless networks that has recently catalyzed attention of the international industrial and academic communities [19] and it can play a key role in the proposed system. Cooperation can have different characteristics and meanings in wireless communications, where cooperating entities can be of different types (sensors, actuators, relief workers' equipment, etc.) and cooperative techniques can be applied within and across the OSI layers, and even take place between heterogeneous networks. Canonical forms of cooperation, like multi-hop relaying, antenna diversity, and cooperative coding, have been introduced so far in the literature. Furthermore, cooperative systems and radios have been recognized as enablers of fourth generation networks [20]. In principle, cooperative communications among users have the potential to enhance key capabilities and performance of wireless communications, such as increasing the data rate, reducing the transfer delay, increasing QoS, limiting power consumption, enhancing spectrum efficiency and capacity. Issues related to MAC protocol design in cognitive radio networks have been considered in the survey of [21], where the differences between the conventional multi-channel MAC protocols and the MAC protocols for opportunistic spectrum access are highlighted. Substantially MAC protocols should become opportunistic as well, able to access unoccupied resources opportunistically and coexist with existing networks, like WLAN [22].

\section{E. Definition of a reconfigurable BTS}

Reconfigurable base stations can provide a convenient choice as the access points in emergency phases. The open source Unix project Open BTS [23-24] (based on the Universal Software Radio Peripheral, USRP [25]) aims to allow the implementation of a GSM air interface BTS, enabling the calls to the GSM handsets, using the Asterisk [26] software PBX to connect calls, even towards external networks. The combination of the GSM air interface and the VoIP backhaul carries to a new type of cellular network. This system can be deployed and operated at a much lower cost than existing technologies, but it will still be compatible with most of the handsets already present in the market. The use of Asterisk, a software implementation of a telephone private branch exchange (PBX), allows inbound and outbound PSTN calls and reduces the complexity of the network since most of the telephone switching functions, calling features and mobility management functions are included in Asterisk itself: indeed the need for most of the network infrastructure (e.g. HLRs, MSCs, etc.) is overcome. A VoIP gateway enables the connection of the cellular network to several types of services and in perspective also to LTE system [27]. 


\section{CONCLUSIONS}

The proposed system defines a viable solution to provide the functionalities of communications, localization and monitoring in emergency situations, where the role of a satellite system is essential. In perspective it can afford improvements and better services with respect to presently available systems in all different emergency contexts. It is coherent with the guidelines for the development of future emergency systems, that aim to help potential implementers and end user to exploit new technologies efficiently and to improve the quality of intervention operations.

\section{REFERENCES}

[1] ETSI Recommendation TS 102181 V.1.1.1 (200512) $[\mathrm{XX}]$, "Emergency Communications (EMTEL); Requirements for communication between authorities/organizations during emergencies".

[2] G. Hazzani, "FP7 -ESS Emergency Support System: Introduction", Sept. 2009, available at: http://www.essproject.eu/downloads/category/2-presentations.html.

[3] SAFER project leaflet, available at: http://www.emergencyresponse.eu/.

[4] M. Berioli, N. Courville, and M. Werner, "Emergency Communications over Satellite: the WISECOM Approach", Proc. of 16th IST Mobile and Wireless Communications Summit, 2007, 1-5 July 2007.

[5] H. Aiache, R. Knopp, K. Koufos, H. Salovuori, P. Simon, "Increasing Public Safety Communications Interoperability: The CHORIST Broadband and Wideband Rapidly Deployable Systems", IEEE International Conference on Communications Workshops, June 2009.

[6] E. Del Re, S. Morosi, S. Jayousi, L. S. Ronga, R. Suffritti, "Satellite role in emergency services", Proc. of the 2nd European Wireless Technology Conference, EuWiT 2009, Rome, Italy, 28-29 Sept. 2009.

[7] SALICE website: http://lenst.det.unifi.it/salice/.

[8] E. Del Re, M. Ruggieri, Eds, Satellite Communications and Navigation Systems, Springer, 2007 (currently under Chinese translation).

[9] S. Voigt, et al. "Satellite image analysis for disaster and crisis management support," IEEE Trans. Geosci. Remote Sens., vol. 45, no. 6, Jun. 2007.

[10] F. Dell'Acqua, D. Polli, S. Ferrari, "Spaceborne support to post-earthquake operations: detection of sparse man-made features in widescale safety inspection", EUSAR 2010, Aachen, Germany, 8-10 June 2010 .

[11] H. Aoki, M. Matsuoka, and F. Yamazaki, "Characteristics of satellite SAR images in the damaged areas due to the Hyogoken-Nanbu earthquake," in Proc. $19^{\text {th }}$ Asian Conf. Remote Sens., 1998, vol. C7.

[12] M. Matsuoka and F. Yamazaki, "Use of satellite SAR intensity imagery for detecting building areas damaged due to earthquakes," Earthquake Spectra, vol.
20, no. 3, 2004.

[13] C. Edery-Guirardo, J. P. Aguttes, E. Bouisson and F. Forestier, "Small Relay Satellites for Improving the Reactivity of Observation Satellites", International Astronautical Federation, Paper IAC-03-B.2.04, Sept. 2003.

[14] W. Colitti, K. Steenhaut, N. Descouvemont, "Satellite-based Wireless Sensor Networks - Global Scale Sensing with Nano- and Pico-Satellites" SenSys'08, November 5-7, 2008, Raleigh, North Carolina, USA.

[15] F. Dominici, G. Marucco, P. Mulassano, A. Defina, K. Charqane, "Navigation In Case of Emergency (NICE): An Integrated NAV/COM Technology for Emergency Management," Consumer Communications and Networking Conference, 5th IEEE CCNC 2008, 10-12 Jan. 2008.

[16] F. Dominici, A. Defina, F. Dovis, "An Augmented GPS/EGNOS Localization System for Alpine Rescue Teams Based on a VHF Communication Infrastructure", Position, Location and Navigation Symposium, 2006 IEEE/ION , April 25-27, 2006.

[17] N. Drawil, O. Basir, "Vehicular Collaborative Technique for Location Estimate Correction", Vehicular Technology Conference, IEEE 68th VTC 2008-Fall, 21-24 Sept. 2008 .

[18] P. Cui, M. Cao, "Collaborative autonomous navigation in unknown environment: Intelligent Control and Automation", WCICA 2008 7th World Congress, 25-27 June 2008.

[19] F.H.P. Fitzek, M. Katz, Cooperation in Wireless Networks: Principles and Applications - Real Egoistic Behavior is to Cooperate!', Springer, 2006.

[20] M. Dohler, D.-E. Meddour, S.-M. Senouci, A. Saadani, 'Cooperation in 4G -Hype or Ripe?', IEEE Technology Society Magazine, Spring 2008, Vol. 27, n.1.

[21] H. Wang, H. Qin, and L. Zhu, "A Survey on MAC Protocols for Opportunistic Spectrum Access in Cognitive Radio Networks", Proc. of 2008 IEEE Int. Conf. on Computer Science and Software Engineering.

[22] S-Y. Hung, E.H-K Wu, and G-H. Chen, "An Opportunistic Cognitive MAC protocol for Coexistence with WLAN", Proc. of IEEE ICC 2008 Conference.

[23] http://openbts.sourceforge.net.

[24] http://gnuradio.org/redmine/wiki/gnuradio/OpenBTS

[25] http://www.ettus.com/

[26] http://www.asterisk.org

[27] 3GPP TR 25.913 "Requirements for E-UTRA and E-UTR. 